\title{
Review: depression, social isolation, and certain life events are associated with the development of coronary heart disease
}

Bunker SJ, Colquhoun DM, Esler MD, et al. Stress and coronary heart disease: psychosocial risk factors. Med J Aust 2003;178:272-6.

\section{QUESTION: Are certain psychosocial risk factors (stressors) associated with the development and progression of coronary heart disease (CHD)?}

Sources of funding:

National Heart

Foundation of Australia

and Beyondblue: the

National Depression

Initiative.

For correspondence:

Dr S J Bunker,

National Heart

Foundation,

Melbourne, Victoria,

Australia.

steve.bunker@

heartfoundation.com.au

\section{Data sources}

Reviews were identified by searching Medline, EMBASE/Excerpta Medica, and PsycINFO using 47 search terms to describe study design, outcomes, and stressors. Reference lists of reviews and personal files of the investigators were also searched.

\section{Study selection}

Reviews were selected if they were published in English in peer reviewed journals after 1979 and included prospective studies with $\geq 100$ patients, and if CHD outcomes included myocardial infarction, myocardial revascularisation, and CHD death.

\section{Data extraction}

2 investigators independently assessed the quality of the selected reviews using a validated 7 point score. For reviews with a score $\geq 4$, data were extracted on years covered, reviewers' backgrounds, stressors examined, number of studies, outcomes, measures of association, results, confounders, features of causality, statistical and clinical significance, and generalisability. The evidence in support of an association was rated as good (clear preponderance of good quality positive reviews over null reviews), fair (small number of good quality positive reviews), and poor (preponderance of good quality null or equivocal reviews or no clear preponderance of positive or negative reviews). When reviews included both prospective and case control studies, only the data pertaining to prospective studies were included.

\section{Main results}

57 reviews met the selection criteria and 15 reviews had a quality score $\geq 4$. 7 stressors were shown to be independent risk factors for CHD (table). Good evidence showed an association with CHD for depression, social isolation or lack of social support, and catastrophic life events. Fair evidence existed for acute life events. The evidence was equivocal for hostility or anger, work characteristics, and anxiety. The evidence did not support an association between panic disorder and CHD.

\section{Conclusion}

Strong evidence supports an association between depression, social isolation or lack of social support, and catastrophic life events and coronary heart disease.
Association of psychosocial risk factors and coronary heart disease*

\begin{tabular}{|c|c|c|c|c|}
\hline \multirow{2}{*}{$\begin{array}{l}\text { Psychosocial risk } \\
\text { factors }\end{array}$} & \multicolumn{3}{|c|}{$\begin{array}{l}\text { Association with CHD } \\
\text { (number of reviews) }\end{array}$} & \multirow[t]{2}{*}{$\begin{array}{l}\text { Association/Strength of } \\
\text { evidence }\end{array}$} \\
\hline & Yes & Equivocal & No & \\
\hline Depression & 4 & & & Yes/Good \\
\hline $\begin{array}{l}\text { Social isolation or lack of } \\
\text { social support }\end{array}$ & 4 & & & Yes/Good \\
\hline Catastrophic life events & 3 & & & Yes/Good \\
\hline Acute life events & 1 & & & Yes/Fair \\
\hline Hostility or anger & 1 & 2 & 1 & Equivocal/Poor \\
\hline Work characteristics & 2 & 1 & & Equivocal/Poor \\
\hline Anxiety & 1 & 1 & 1 & Equivocal/Poor \\
\hline Panic disorder & & & 1 & No/Poor \\
\hline
\end{tabular}

*Good evidence was a clear preponderance of good quality, positive reviews over null reviews; fair evidence was a small number of such reviews; poor evidence was a preponderance of good quality null or equivocal reviews or no clear preponderance of positive or negative reviews.

\section{COMMENTARY}

Consensus committees are a critical element in the chain of basic research leading to improved clinical practice. The review by Bunker $e t$ al is well researched and written and provides clear conclusions. Although "stress" is widely understood, it is a fuzzy concept and the authors focus on better defined psychological constructs that represent emotional distress. However, they do not discriminate between individual characteristics (eg, anxiety or depression) and objective environmental features (eg, life events or job control). This distinction is critical because the environmental stimuli are often impossible to change.

The authors reviewed previously published (1960-2001) review articles. Comprehensiveness (a strength) can result from this strategy, but there also is a cost; if the most recent review was published in 2001, it means that the past 4-5 years of published research were not considered in this 2003 article because of cumulative publication lag. On the whole, it is not a major problem for trustworthiness of the conclusions given the known consistency in research linking depression and social isolation to CHD.

What do the results mean clinically? Importantly, the most salient distress variables have a risk ratio similar in size to biological risk factors (hypertension and dyslipidaemia) with a 3-4 fold relative risk. Unfortunately for patients, we lack consensus on how to screen for emotional distress, and few insurance carriers pay for treating it despite impressive data about effectiveness. Bunker et al's conclusions challenge us to swiftly find consensus on psychological screening tools and to aggressively market nondrug distress treatment to third-party payers.

Wolfgang Linden, $\mathrm{PhD}$ University of British Columbia Vancouver, British Columbia, Canada 